\title{
Observation of the $Z_{c s}(3985)^{-}$and studies on the $Z_{c}$ states at BESIII
}

\section{Ziyi Wang*}

University of Chinese Academy of Sciences, Yuquan Road, Beijing, P.R.China

E-mail: wangziyi181@mails.ucas.ac.cn

BESIII has announced an observation of the structure at the kinematical threshold in the $D_{s}^{-} D^{* 0}\left(D_{s}^{*-} D^{0}\right)$ mass distribution, which is interpreted as a tetraquark candidate, called $Z_{c s}(3985)^{-}$. This is the first candidate for a tetraquark meson containing hidden-charm with non-zero strangeness. In addition, BESIII has been devoting on the studies on the nonstrange charmoniumlike $Z_{c}$ states for years and produced many interesting results. We report the discovery of the $Z_{c s}(3985)^{-}$and the progress of studies on the $Z_{c}$ states.

10th International Workshop on Charm Physics (CHARM2020),

31 May - 4 June, 2021

Mexico City, Mexico - Online

\footnotetext{
${ }^{*}$ Speaker (On behalf of the BESIII Collaboration)
} 


\section{Introduction}

The $X Y Z$ mesons are an assortment of recently discovered resonance-like structures in hadronic final states that contain either a $c$ and $\bar{c}$, or a $b$ and $\bar{b}$ quark pair, with properties that do not match to expectations for any of the currently unassigned $c \bar{c}$ charmonium or $b \bar{b}$ bottomonium states. It provides unique access to understand the non-perturbative effect and color confinement in the QCD theory. In this proceeding, we focus on the isovector $Z$ states. Since they are known to contain an isosinglet $c \bar{c}$ pair, they must also contain light quarks to account for the non-zero isospin. One of the first of these states to be observed, the $Z_{c}$ (3900), was discovered by BESIII [1]. And then there is a series of results reported by BESIII, like the charged and neural $Z_{c}$ (3900) and $Z_{c}$ (4020) [2-8], and the spin analysis of charged $Z_{c}$ (3900) [9]. In 2021, BESIII firstly reported a tetraquark candidate containing hidden-charm with non-zero strangeness, $Z_{c s}$ [10], which will be introduced later in this text along with other recently $Z_{c}$ results on BESIII.

The BESIII detector and BEPCII accelerator play a important role in the study of $X Y Z$ states. The BEPCII collider is a double-ring multi-bunch collider with a design luminosity of $1 \times 10^{33} \mathrm{~cm}^{-2} \mathrm{~s}^{-1}$ optimized at a center-of-mass energy of $2 \times 1.89 \mathrm{GeV}$ and providing $e^{+} e^{-}$beams in the energy range of $\sqrt{s}=2 \sim 4.95 \mathrm{GeV}$. For $X Y Z$ study, a data sample corresponding to an integrated luminosity of $21.9 \mathrm{fb}^{-1}$ was recorded at $\sqrt{s}$ from 3.8 to $4.95 \mathrm{GeV}$ in the last decade. This provided an excellent platform for the study of $X Y Z$ physics.

\section{Observation of $Z_{c s}(3985)^{-}$}

In electron-positron annihilation, the charged $Z_{c}(3900)$ and $Z_{c}(4020)$, as well as their neutral partners, have been observed at BESIII [1-8], Belle [11], and CLEO experiments [12] in a variety of decay modes. Assuming SU(3) flavor symmetry, one would expect the existence of strange partners to the $Z_{c}$, denoted as $Z_{c s}$, with quark content $c \bar{c} s \bar{q}(\bar{q}=\bar{u}, \bar{d})$. There were no signs for $Z_{c s}$ states in experiment until BESIII firstly reported the measurement in 2021 [10].

With a total integrated luminosity of $3.7 \mathrm{fb}^{-1}$ at center-of-mass energies $\sqrt{s}=4.628 \sim$ $4.698 \mathrm{GeV}$, BESIII investigate the processes $e^{+} e^{-} \rightarrow K^{+} D_{s}^{-} D^{* 0}$ and $K^{+} D_{s}^{*-} D^{0}$ by using a novel partial reconstruction method that relies on the detection of charged Kaon and oppositely charged $D_{s}$ meson. After reducing the combinatorial backgrounds, a clear enhancement is seen near the $D_{s}^{-} D^{* 0}$ and $D_{s}^{*-} D^{0}$ mass thresholds. And a intensive study on the high-excited $D_{(s)}^{* *}$ are preformed with control sample studies and amplitude analysis method. None of them can explain the enhancement. Furthermore, by using the method of amplitude construction, we also exclude the interference effect among the known $D_{(s)}^{* *}$ excited states.

Since the known charmed mesons and combinatorial backgrounds cannot explain the excess, it matches a hypothesis of a $D_{s}^{-} D^{* 0}$ and $D_{s}^{*-} D^{0}$ resonant structure $Z_{c s}(3985)^{-}$with a mass-dependentwidth Breit-Wigner line shape well; a fit gives the resonance mass of $\left(3985.2_{-2.0}^{+2.1} \pm 1.7\right) \mathrm{MeV} / c^{2}$ and width of $\left(13.8_{-5.2}^{+8.1} \pm 4.9\right) \mathrm{MeV}$, as shown in Fig. 1. This corresponds to a pole position as

$$
\begin{aligned}
m_{\text {pole }}\left[Z_{c s}(3985)^{-}\right] & =\left(3982.5_{-2.6}^{+1.8} \pm 2.1\right) \mathrm{MeV} / c^{2} \\
\Gamma_{\text {pole }}\left[Z_{c s}(3985)^{-}\right] & =\left(12.8_{-4.4}^{+5.3} \pm 3.0\right) \mathrm{MeV}
\end{aligned}
$$




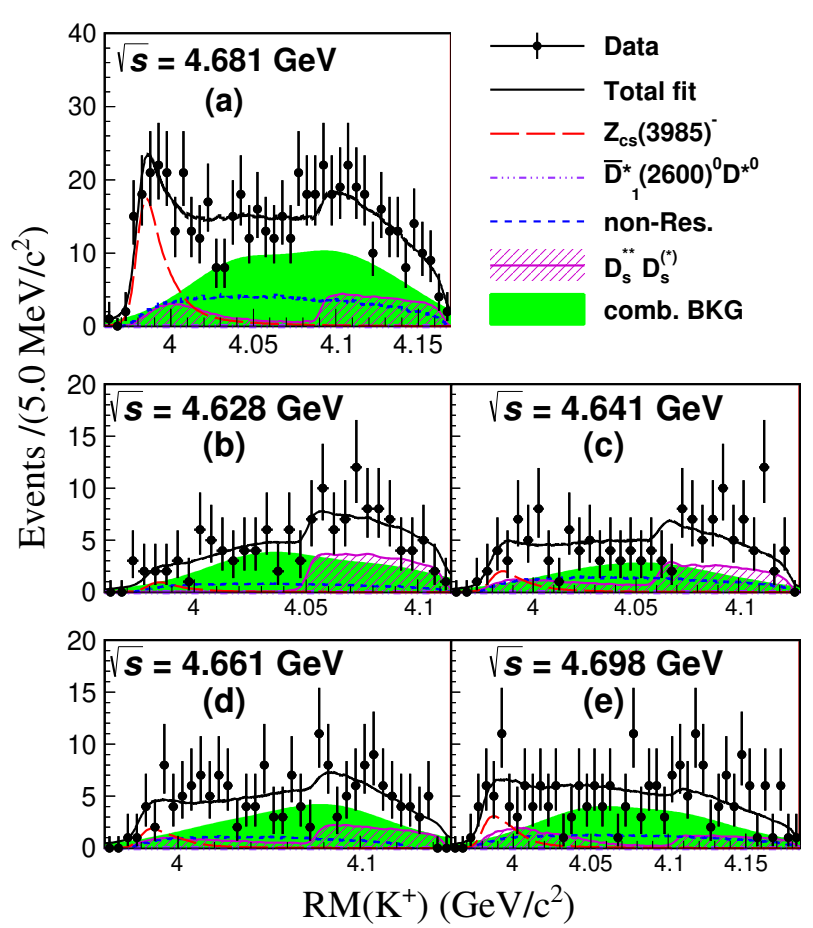

Figure 1: Simultaneous unbinned maximum likelihood fit to the $K^{+}$recoil-mass spectra in data at $\sqrt{s}=$ $4.628 \sim 4.698 \mathrm{GeV}$.

The significance of the resonance hypothesis is estimated to be $5.3 \sigma$ over the pure contributions from the conventional charmed mesons.

A few months after the BESIII's result, LHCb Collaboration announces their observation of $Z_{c s}(4000)$ in $B^{+} \rightarrow J / \psi \phi K^{+}$process, with a mass of $4003 \pm 6_{-14}^{+4} \mathrm{MeV}$ and a width of $131 \pm 15 \pm$ $26 \mathrm{MeV}$ [13]. The discussion on the relationship between these two two $Z_{c s}$ states has been going on to this day [14-17]. Since the masses of these two $Z_{c s}$ states are quite close, they could be the same state. However, given about their difference on width, they should be different states. In this case, it would imply that there should be a wide state with mass close to that of the narrow $Z_{c}(3900)$ as the $\mathrm{SU}(3)$ partner of $Z_{c s}(4000)$.

Up to now, no firm conclusion has yet been reached and it deserves more dedicated effort to pin down the relationship of these two $Z_{c s}$ states. More BESIII data spanning the $4.68 \mathrm{GeV}$ energy region is needed to thoroughly explore the $Z_{c s}$ production mechanism, measure its spin-parity quantum numbers, and search for its neutral $Z_{c s}^{0}$ counterpart as well as high-excited $Z_{c s}^{\prime}$ states.

\section{Study of $e^{+} e^{-} \rightarrow \pi^{0} \pi^{0} J / \psi$ and $Z_{c}(3900)^{0}$}

The neutral $Z_{c}(3900)^{0}$ was shown an evidence on CLEO-c in process $e^{+} e^{-} \rightarrow \pi^{0} \pi^{0} J / \psi[12]$ and observed by BESIII via $e^{+} e^{-} \rightarrow \pi^{0} \pi^{0} J / \psi$ and $e^{+} e^{-} \rightarrow \pi^{0}\left(D \bar{D}^{*}\right)^{0}$ reactions [18, 19]. To investigate more about $Z_{c}(3900)^{0}$ properties, using $12.4 \mathrm{fb}^{-1}$ data samples, an amplitude analysis of $e^{+} e^{-} \rightarrow \pi^{0} \pi^{0} J / \psi$ is performed [20]. In the nominal fit, the intermediate process $e^{+} e^{-} \rightarrow$ $\sigma J / \psi, f_{0}(980) J / \psi, f_{0}(1370) J / \psi$, and $\pi^{0} Z_{c}(3900)^{0}$ are included. Based on the simultaneous partial wave analysis fit results, as shown in Fig. 2, the $\pi^{0} \pi^{0} S$-wave contribution dominates. The 
spin-parity of $Z_{c}(3900)^{0}$ is determined to be $J^{P}=1^{+}$with a statistical significance of more than $9 \sigma$, and the mass and width are measured to be $(3893.0 \pm 2.3 \pm 19.9) \mathrm{MeV} / c^{2}$ and $(44.2 \pm 5.4 \pm 9.1) \mathrm{MeV}$, respectively. These values are consistent with those of the charged $Z_{c}(3900)^{ \pm}$observed in $e^{+} e^{-} \rightarrow$ $\pi^{+} \pi^{-} J / \psi$.
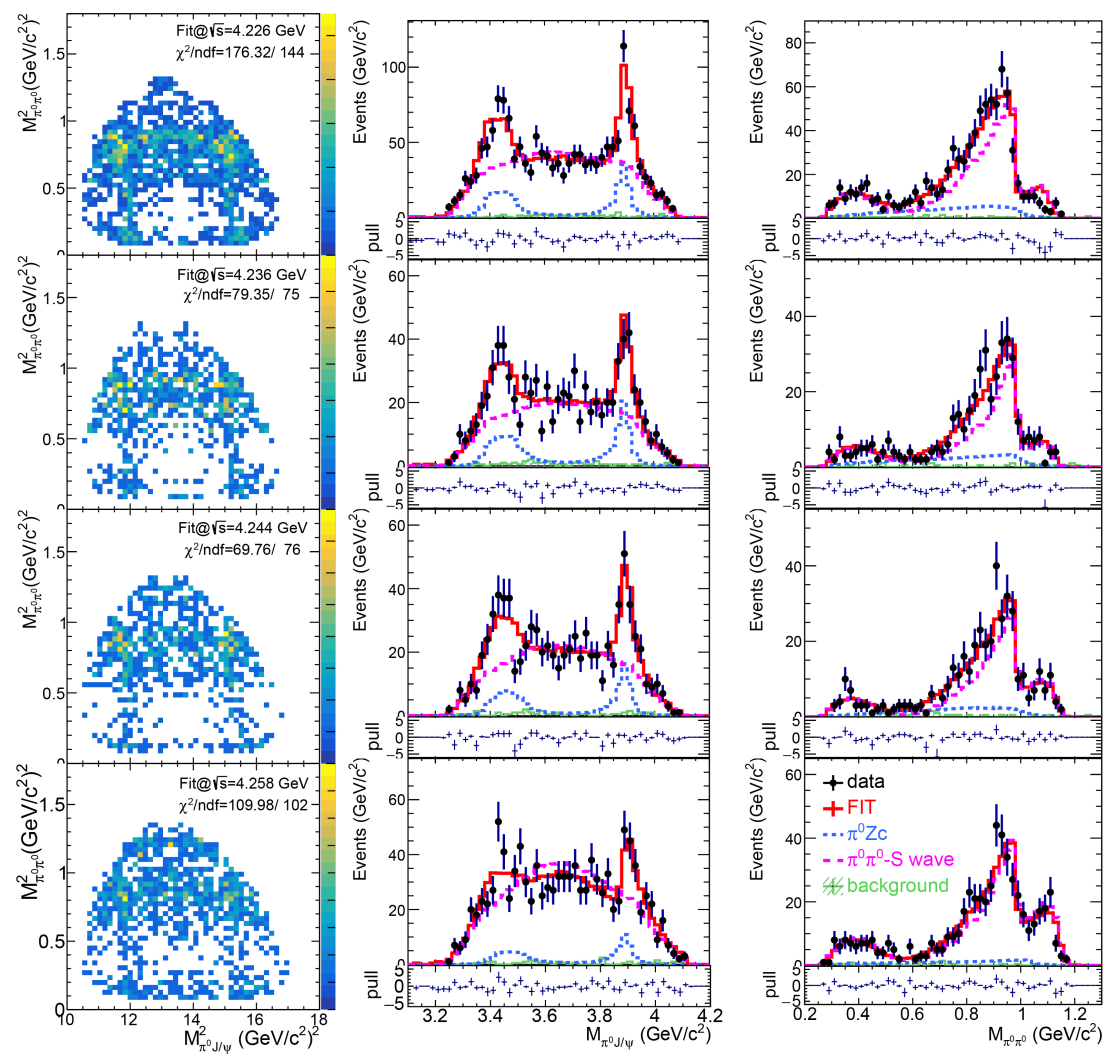

Figure 2: (Left column) Dalitz plots of $M_{\pi^{0} J / \psi}^{2}$ versus $M_{\pi^{0} \pi^{0}}^{2}$, invariant-mass projections (middle column) $M_{\pi^{0} J / \psi}$ and (right column) $M_{\pi^{0} \pi^{0}}$ of the results of the nominal PWA for data samples $\sqrt{s}=4.226 \sim$ $4.258 \mathrm{GeV}$. Points with errors are data, red solid curves are the total fit results, the blue dashed (magenta long-dashed) curves represent $Z_{c}(3900)^{0}\left(\pi^{0} \pi^{0}\right.$-S wave) components, and green shaded histograms represent the estimated backgrounds. Each event appears twice in the Dalitz plots and $M_{\pi^{0} J / \psi}$ distributions. The $\chi^{2} /$ ndf is calculated by merging those bins with less than 10 events in the Dalitz plots.

\section{Search for $e^{+} e^{-} \rightarrow \chi_{c J} \pi^{+} \pi^{-}$and a charmoniumlike structure decaying to $\chi_{c J} \pi^{ \pm}$}

$Z_{c 1}(4050)^{+}$and $Z_{c 2}(4250)^{+}$were firstly observed by the Belle Collaboration in the final states of $\chi_{c 1} \pi$ via the process $\bar{B}^{0} \rightarrow K^{-} \pi^{+} \chi_{c 1}$ with significance both greater than $5 \sigma$ [21]. However, the BABAR Collaboration performed a similar research via $\bar{B}^{0} \rightarrow K^{-} \pi^{+} \chi_{c 1}, B^{+} \rightarrow K_{S}^{0} \pi^{+} \chi_{c 1}$ but obtained a negative result [22].

With the data sets collected at $\sqrt{s}=4.18 \sim 4.60 \mathrm{GeV}$, BESIII try to verify the existence of the two $Z_{c}$ states [23]. Based on a total integral luminosity at $11.2 \mathrm{fb}^{-1}$, no significant signal for the processes $e^{+} e^{-} \rightarrow \chi_{c J} \pi^{+} \pi^{-}(J=0,1,2)$ has been observed. In this case, we provide the upper limits for the three channels at each energy point, as shown in Fig. 3. 

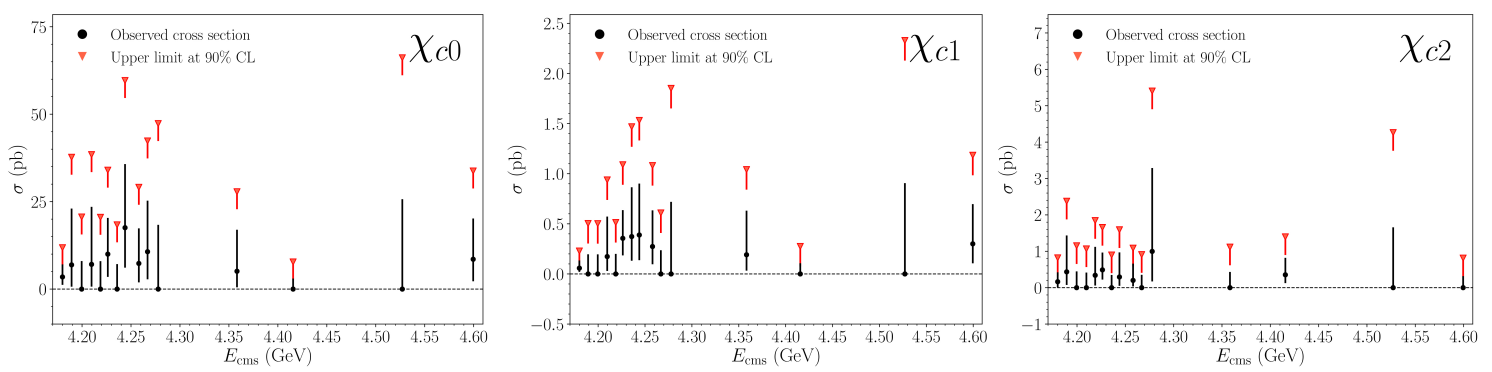

Figure 3: Cross section (black) and corresponding upper limit (red) for the reaction channels $e^{+} e^{-} \rightarrow$ $\chi_{c J} \pi^{+} \pi^{-}$versus the center-of-mass energy $E_{\mathrm{cms}}$.

\section{Search for a $Z_{c}$ state decaying to $\eta_{c} \pi$}

Inspired by the evidence of $Z_{c}(4100)^{+} \rightarrow \pi^{+} \eta_{c}$ via $\bar{B}^{0} \rightarrow K^{-} Z_{c}(4100)^{+}$process from $\mathrm{LHCb}$ Collaboration in 2018 [24], three different exclusive reactions $e^{+} e^{-} \rightarrow \eta_{c} \pi^{+} \pi^{-} \pi^{0}, e^{+} e^{-} \rightarrow \eta_{c} \pi^{+} \pi^{-}$, and $e^{+} e^{-} \rightarrow \eta_{c} \pi^{0} \gamma$ are measured by BESIII [25]. The reaction $e^{+} e^{-} \rightarrow \eta_{c} \pi^{+} \pi^{-} \pi^{-}$is observed for the first time with an energy-dependent Born cross section measured to be consistent with the production via the intermediate $Y(4260)$ resonance. The largest observed cross section of measured at $\sqrt{s}=4.23 \mathrm{GeV}$ also has the highest significance value of $4.1 \sigma$. Summing up all six cross sections values of center-of-mass energies from 4.18 to $4.60 \mathrm{GeV}$, the significance reduce to $5.2 \sigma$.

Based on the energy points at $\sqrt{s}=4.18 \sim 4.60 \mathrm{GeV}$, corresponding to a integral luminosity as $7.3 \mathrm{fb}^{-1}$, we perform a search for a charmoniumlike $Z_{c}$ state that decays to $\eta_{c} \pi$. No significant signal observed and the corresponding upper limits on the cross section of $\eta_{c}$ and $Z_{c}$ production are provided, as shown in Fig. 4.
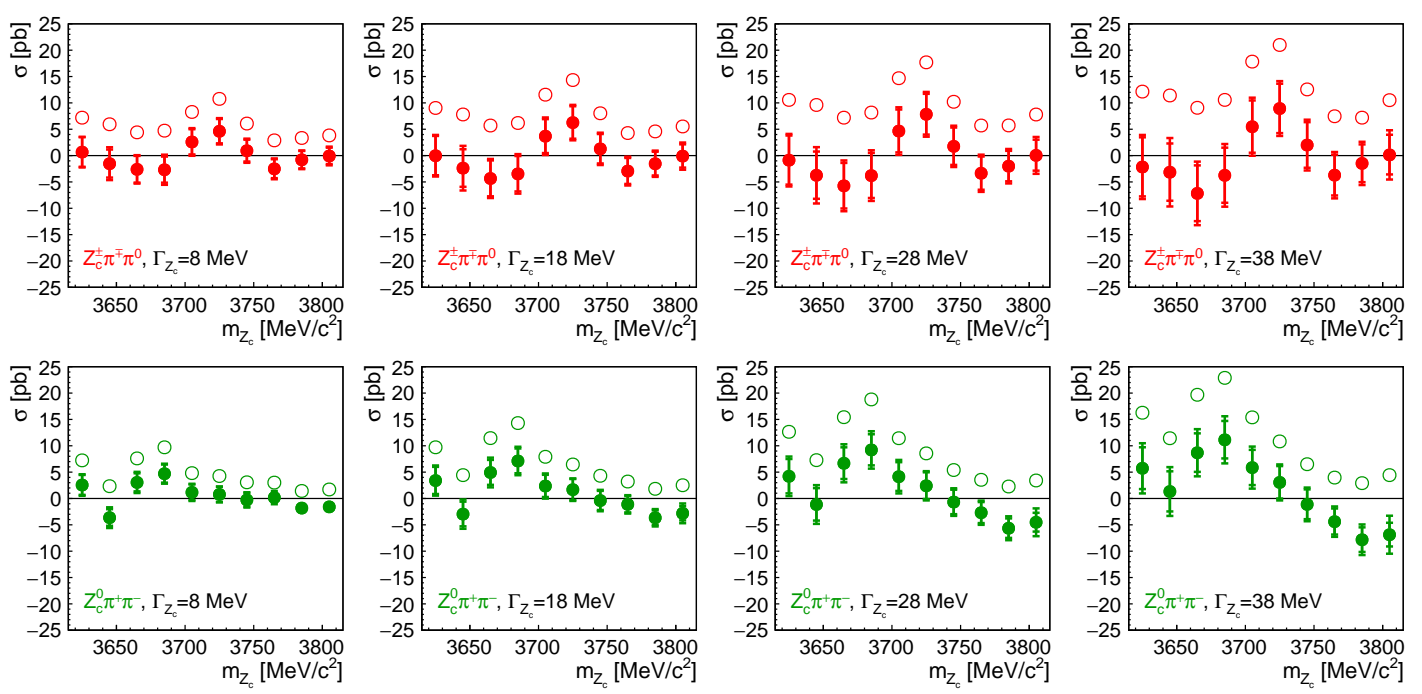

Figure 4: Search for a possible charged (top) or neutral (bottom) $Z_{c}$ state in the vicinity of the $D \bar{D}$ threshold, decaying to $\eta_{c} \pi$ in $e^{+} e^{-} \rightarrow \eta_{c} \pi^{0} \pi^{+} \pi^{-}$reactions. Shown are the results of mass and width scans in terms of the measured $\left(m_{Z_{c}}, \Gamma_{Z_{c}}\right)$-dependent cross section $\sigma$ (red/green data points) together with the upper limits (open circles) on the Born cross section $\sigma_{\text {Born }}$. The measurements have been performed for four assumed widths $\Gamma_{Z_{c}}=8,18,28,38 \mathrm{MeV}$ and ten assumed masses $m_{Z_{c}}=3625 \sim 3805 \mathrm{MeV} / c^{2}$ at $\sqrt{s}=4.23 \mathrm{GeV}$. 


\section{Study of $e^{+} e^{-} \rightarrow \pi^{0} X(3872) \gamma$ and search for $Z_{c}(4020)^{0} \rightarrow X(3872) \gamma$}

Figuring out the possible connection between $Z$ and $X$ states in experiment could be a clue to facilitate a better theoretical interpretation. One such connection [26] could be a transition $Z_{c}(4020)^{0} \rightarrow X(3872) \gamma$ in the scenario where the $X(3872)$ is dominantly an $S$-wave $D^{0} \bar{D}^{* 0}$ molecule and the $Z_{c}(4020)^{0}$ is an isotopic triplet of near-threshold S-wave $D^{0} \bar{D}^{* 0}$ resonances. Therefore, search for the transition $Z_{c}(4020)^{0} \rightarrow X(3872) \gamma$ can help to quantitatively study the molecular picture of the $X(3872)$.

BESIII report the search for the reaction $e^{+} e^{-} \rightarrow \pi^{0} X(3872) \gamma$ and $Z_{c}(4020)^{0} \rightarrow X(3872) \gamma$ based on the data in range of $\sqrt{s}=4.18 \sim 4.60 \mathrm{GeV}$ [27]. In neither of the two processes are significant signals observed. Upper limit at $90 \%$ C.L. on the cross sections multiplied by the branching fractions, $\sigma\left(e^{+} e^{-} \rightarrow \pi^{0} X(3872) \gamma\right) \cdot \mathcal{B}\left(X(3872) \rightarrow \pi^{+} \pi^{-} J / \psi\right)$ and $\sigma\left(e^{+} e^{-} \rightarrow\right.$ $\left.\pi^{0} Z_{c}(4020)^{0}\right) \cdot \mathcal{B}\left(Z_{c}(4020)^{0} \rightarrow X(3872) \gamma\right)$, are reported for each energy point, as shown in Fig. 5. The measured results of the process $e^{+} e^{-} \rightarrow \pi^{0} X(3872) \gamma$ are not in conflict with the theoretical expectation of about $0.1 \mathrm{fb}^{-1}$ [28]. Using the experimental results on the $\sigma\left(e^{+} e^{-} \rightarrow\right.$ $\left.\pi^{0} Z_{c}(4020)^{0}\right) \cdot \mathcal{B}\left(Z_{c}(4020)^{0} \rightarrow\left(D^{*} \bar{D}^{*}\right)^{0}\right.$ at $\sqrt{s}=4.226$ and $4.258 \mathrm{GeV}$ [29], the ratio

$$
\frac{\mathcal{B}\left(Z_{c}(4020)^{0} \rightarrow X(3872) \gamma \cdot \mathcal{B}\left(X(3872) \rightarrow \pi^{+} \pi^{-} J / \psi\right)\right.}{\mathcal{B}\left(Z_{c}(4020)^{0} \rightarrow\left(D^{*} \bar{D}^{*}\right)^{0}\right)}
$$

is determined to be less than $0.15 \%$ at the $90 \%$ C.L. The ratio does not contradict the prediction reported in Ref. [26] based on the molecular picture.
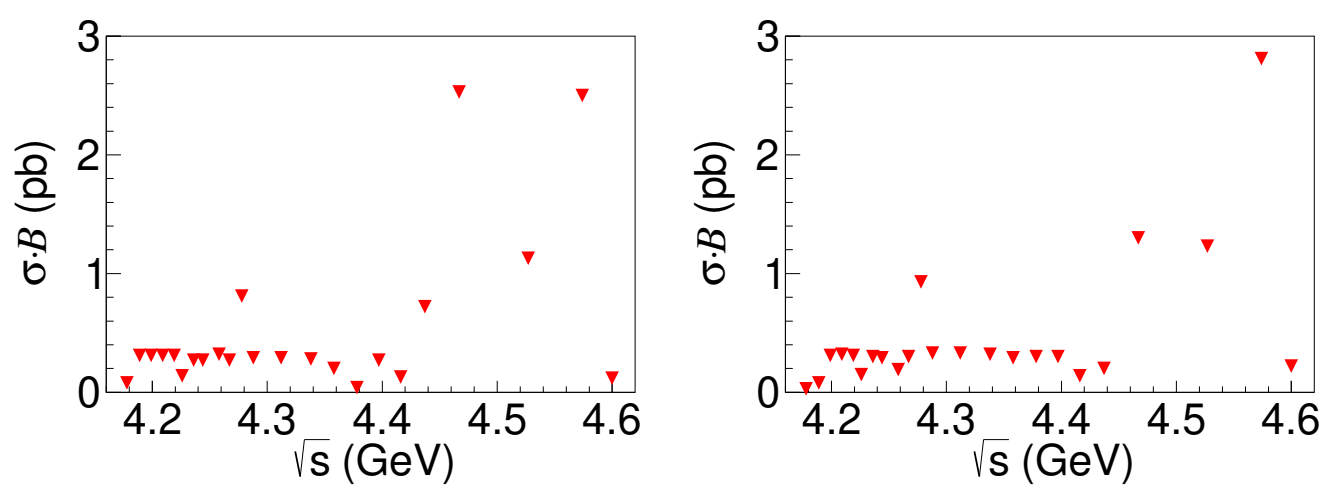

Figure 5: The upper limits at the $90 \%$ C.L. on $\sigma\left(e^{+} e^{-} \rightarrow \pi^{0} X(3872) \gamma\right) \cdot \mathcal{B}\left(X(3872) \rightarrow \pi^{+} \pi^{-} J / \psi\right)$ (left) and $\sigma\left(e^{+} e^{-} \rightarrow \pi^{0} Z_{c}(4020)^{0}\right) \cdot \mathcal{B}\left(Z_{c}(4020)^{0} \rightarrow X(3872) \gamma\right) \cdot \mathcal{B}\left(X(3872) \rightarrow \pi^{+} \pi^{-} J / \psi\right)$ (right) for each energy point.

\section{Summary}

Over the past decade, we've seen a rocketing growth market of $X Y Z$ states. It provides a promising blueprint for both theory and experiment. BESIII made a unique contribution, like $Z_{c}(3900)$ and $Z_{c s}(3985)$ and so on, and will continue its exploration in $X Y Z$ studies. One can look forward to the future upgraded BEPCII machine [30]. The mass-of-center energy could reach 
$5.6 \mathrm{GeV}$, and the luminosity can be three times as higher as the current state. With the upgraded machine, much more programs and more dedicated effort will promote our understanding on the $X Y Z$ physics.

\section{References}

[1] M. Ablikim et al. (BESIII Collaboration), Phys. Rev. Lett. 110, 252001 (2013).

[2] M. Ablikim et al. (BESIII Collaboration), Phys. Rev. Lett. 111, 242001 (2013).

[3] M. Ablikim et al. (BESIII Collaboration), Phys. Rev. Lett. 112, 022001 (2014).

[4] M. Ablikim et al. (BESIII Collaboration), Phys. Rev. Lett. 112, 132001 (2014).

[5] M. Ablikim et al. (BESIII Collaboration), Phys. Rev. Lett. 113, 212002 (2014).

[6] M. Ablikim et al. (BESIII Collaboration), Phys. Rev. Lett. 115, 112003 (2015).

[7] M. Ablikim et al. (BESIII Collaboration), Phys. Rev. Lett. 115, 182002 (2015).

[8] M. Ablikim et al. (BESIII Collaboration), Phys. Rev. Lett. 115, 222002 (2015).

[9] M. Ablikim et al. [BESIII], Phys. Rev. Lett. 119, 072001 (2017)

[10] M. Ablikim et al. (BESIII Collaboration), Phys. Rev. Lett. 126, 102001 (2021).

[11] Z. Q. Liu et al. (Belle Collaboration), Phys. Rev. Lett. 110, 252002 (2013) [erratum: Phys. Rev. Lett. 111, 019901 (2013)].

[12] T. Xiao, S. Dobbs, A. Tomaradze and K. K. Seth, Phys. Lett. B 727, 366 (2013).

[13] R. Aaij et al. (LHCb Collaboration), Phys. Rev. Lett. 127, 082001 (2021).

[14] Z. Yang, X. Cao, F. K. Guo, J. Nieves and M. P. Valderrama, Phys. Rev. D 103, 074029 (2021).

[15] L. Meng, B. Wang, G. J. Wang and S. L. Zhu, [arXiv:2104.08469 [hep-ph]].

[16] P. G. Ortega, D. R. Entem and F. Fernandez, Phys. Lett. B 818, 136382 (2021).

[17] L. Maiani, A. D. Polosa and V. Riquer, Symmetry 13, 751 (2021).

[18] M. Ablikim et al. (BESIII Collaboration), Phys. Rev. Lett. 115, 112003 (2015).

[19] M. Ablikim et al. (BESIII Collaboration), Phys. Rev. Lett. 115, 222002 (2015).

[20] M. Ablikim et al. (BESIII Collaboration), Phys. Rev. D 102, 012009 (2020).

[21] R. Mizuk et al. (Belle Collaboration), Phys. Rev. D 78, 072004 (2008).

[22] J. P. Lees et al. (BaBar Collaboration), Phys. Rev. D 85, 052003 (2012).

[23] M. Ablikim et al. (BESIII Collaboration), Phys. Rev. D 103, 052010 (2021). 
[24] R. Aaij et al. (LHCb Collaboration), Eur. Phys. J. C 78, 1019 (2018).

[25] M. Ablikim et al. (BESIII Collaboration), Phys. Rev. D 103, 032006 (2021).

[26] M. B. Voloshin, Phys. Rev. D 99, 054028 (2019).

[27] M. Ablikim et al. (BESIII Collaboration), Phys. Rev. D 104, 012001 (2021).

[28] S. Sakai, H. J. Jing and F. K. Guo, Phys. Rev. D 102, 114041 (2020).

[29] M. Ablikim et al. (BESIII Collaboration), Phys. Rev. Lett. 115, 182002 (2015).

[30] M. Ablikim et al. (BESIII Collaboration), Chin. Phys. C 44, 040001 (2020). 